\title{
Adjuvante Therapie von arterieller Hypertonie durch hochfrequentes Blutdruckbiofeedback
}

\author{
Christoph Piesbergen $^{\mathrm{a}} \quad$ Katharina Kircher $^{\mathrm{b}} \quad$ Reinhard Weber $^{\mathrm{c}}$ \\ a Department Psychologie - Klinische Psychologie und Psychotherapie, Ludwig-Maximilians-Universität München, \\ b Gerontopsychiatrischer Dienst für den Landkreis Mühldorf/Inn, \\ ' Klinik Höhenried für Herzkreislaufkrankheiten, Orthopädie und Psychosomatik, gGmbH der Deutschen Rentenversicherung Bayern Süd, \\ Bernried am Starnberger See, Deutschland
}

\author{
Schlüsselwörter \\ Biofeedback - Arterieller Blutdruck - Essentielle \\ Hypertonie $\cdot$ Kontinuierliche Messung
}

\section{Zusammenfassung}

Hintergrund: Der Mangel an praxisnahen Studien zur Wirksamkeit von kontinuierlichem Blutdruckbiofeedback führte zur Entwicklung und Erprobung einer Aufzeichnungs- und Feedbacksoftware, die eine Beat-to-Beat-Darstellung des arteriellen Blutdrucks auf einem Computermonitor ermöglicht. In Biofeedbacksitzungen mit diesem System machen Hochdruckpatienten die Erfahrung, dass sie über eigene Ressourcen für eine klinisch relevante Blutdrucksenkung verfügen. Patienten und Methoden: Zur Evaluation des Verfahrens wurde an der Klinik Höhenried am Starnberger See (Deutschland) eine Pilotstudie mit 84 stationären Hypertoniepatienten durchgeführt. Zwei Experimentalgruppen, die das normale Heilbehandlungsprogramm der Klinik und zusätzlich 2 (20 Probanden) bzw. 3-6 (22 Probanden) jeweils 30-minütige Biofeedbackbehandlungen erhielten, wurden mit zwei Kontrollgruppen verglichen (20 Probanden mit Pseudobiofeedback und 22 Probanden, die nur das Klinikprogramm durchliefen). Ergebnisse: Aus den mittels Armmanschette gemessenen RR-Werten wurden bei allen Gruppen die Durchschnittswerte des medizinischen Mitteldrucks (MAP) in der ersten und in der Entlassungswoche berechnet und es wurde ein Differenzwert gebildet. Beide Experimentalgruppen erzielten signifikant höhere Senkungen des MAP als die Kontrollgruppen $(p=0,001)$, allerdings schnitt die Experimentalgruppe mit 3-6 Feedbacksitzungen nicht signifikant besser ab als die Gruppe mit nur 2 Sitzungen ( $p=0,517)$. Schlussfolgerungen: Schon eine 2-malige Behandlung mit hochfrequentem Biofeedback kann klinisch relevante Effekte haben, was Hinweise auf einen erheblichen Einfluss von kognitiven Prozessen wie Selbstwirksamkeit und Selbstkontrolle liefert.

\section{Key Words}

Biofeedback - Arterial blood pressure .

Essential hypertension - Continuous registration

\section{Summary}

Beat-to-Beat Blood Pressure Biofeedback as a

Supplementary Treatment for Arterial Hypertension

Introduction: A lack of studies on the effects of continuous blood pressure biofeedback led to the development of a screening and feedback software allowing for a 'beat-to-beat' representation of arterial blood pressure. Through this method patients find that they can exert some control of their blood pressure. Patients and Methods: For evaluation purposes a pilot study was conducted with 84 hypertensive patients at the Klinik Höhenried, Germany. Two experimental groups which received the normal treatment program of the hospital and an additional 2 (20 patients) or 3-6 (22 patients) biofeedback sessions, respectively, were compared with one control group treated additionally with pseudo biofeedback (20 patients) and another control group (22 patients) which underwent the clinic program without biofeedback. Results: Both experimental groups yielded significantly more markedly blood pressure reductions (MAP) than the controls $(p=0.001)$, but patients who received 3-6 feedback sessions did not perform significantly better than patients who received only 2 sessions ( $p=0.517$ ). Conclusions: 2 sessions of feedback treatment can already have clinically relevant effects. This suggests a major influence of cognitive processes such as self-effectiveness and self-control.

\begin{tabular}{ll}
\hline KARGER & $\oplus$ 2008 S. Karger GmbH, Freiburg \\
Fax +497614520714 & Accessible online at: \\
$\begin{array}{l}\text { E-mail Information@Karger.de } \\
\text { www.karger.com }\end{array}$ & www.karger.com/ver
\end{tabular}




\section{Hintergrund}

Die essentielle Hypertonie zählt zu den klassischen psychosomatischen Krankheiten. Die Bezeichnung «essentiell» verdeutlicht, dass keine erkennbare Ursache der Erkrankung bekannt ist; dies ist in 80-90\% der diagnostizierten Hypertonien der Fall [Köhler, 1989]. Derzeit steht zwar ein differenziertes Angebot wirksamer antihypertensiver Medikamente zur Verfügung. Allerdings greifen pharmakologische Therapien nur bei der Symptomatik an, beseitigen jedoch nicht die Ursachen, was zu oft lebenslanger Medikamenteneinnahme, Dosiserhöhung und Gefahr von Rezidiven führen kann. Angesichts der Nebenwirkungen und unerwünschten Begleiterscheinungen können auch therapiebedingte Verschlechterungen im Befinden bzw. der Lebensqualität auftreten [Schandry, 1994]. In der Folge mindert sich sehr oft die Compliance der Patienten, d.h. die Medikamente werden nicht mehr regelmäßig eingenommen oder eigenmächtig abgesetzt. Nebenwirkungsfreie und eventuell kausale Therapiemöglichkeiten stellen somit ein großes Desiderat für die Therapie aller Hypertoniearten dar. Dabei sind z.B. Entspannungstechniken, die immer auch zu einer generellen sympatholytischen Beruhigung führen, hilfreich, denn sie ermöglichen es dem Klienten, einen aktuellen Blutdruckanstieg zu dämpfen oder abzukürzen. Entsprechend angelegte verhaltenstherapeutische Programme sind somit vielversprechend. Ein derartiges Programm, beispielsweise von Kallinke et al. [1982], könnte auch durch die Anwendung instrumenteller Techniken sowohl diagnostisch als auch therapeutisch unterstützt werden.

Solch eine instrumentelle Technik ist das verhaltenspsychologisch orientierte Verfahren des Biofeedback. Dabei handelt es sich um eine Methode, bei der biologische Vorgänge, die nicht oder nur schwer wahrnehmbar sind, in gut wahrnehmbare optische und/oder akustische Signale umgewandelt und somit der bewussten Wahrnehmung durch systematisches Feedback zugänglich gemacht werden [Birbaumer und Schmidt, 1990]. Durch diese Bewusstmachung kann ein gewisses Maß an willentlicher Beeinflussung und Kontrolle aller messbaren Körperfunktionen erlernt werden [Schandry, 1996]. Neben der Selbstkontrolle («self-mastery»: Meichenbaum [1976]) ist der Aufbau einer Selbstwirksamkeitsüberzeugung ein wichtiges Kriterium zur Aufrechterhaltung von durch Biofeedback erreichten Effekten («perceived self-efficacy»: Bandura [1977]). Nur so kann eine unter kontrollierten Übungsbedingungen erreichte psychophysiologische Entspannung allmählich auch unter belastenden Bedingungen wirksam eingesetzt werden. Zwar erbrachte bzw. erbringt die pharmakologische Therapie, wenn sie als einzige Maßnahme gegen Bluthochdruck verwendet wird, immer noch die stärksten Effekte [Andrews et al., 1982; Luborsky et al., 1982], allerdings hat sich schon früh die Erkenntnis durchgesetzt, dass eine Kombination von Pharmako- und Verhaltenstherapie bei Weitem mehr Vorteile bringt, als eine alleinige medikamentöse Behandlung [Vaitl, 1982; Weiss, 1980]. Der Einsatz von Biofeedback wird daher meist bei mildem Hochdruck, bei sehr motivierten Patienten oder zusätzlich zur medikamentösen Behandlung empfohlen.

Eine metaanalytische Studie aus jüngerer Zeit [Yucha et al., 2001] berichtet von Blutdrucksenkungen durch Biofeedback von im Mittel 6,7 $\mathrm{mm} \mathrm{Hg}$ systolisch und 4,9 $\mathrm{mm} \mathrm{Hg}$ diastolisch. Hierbei wurde unter anderem auch darauf geachtet, dass nur Studien mit randomisierten Stichproben und erwachsenen Patienten mit Hypertonie der Stadien I und II (d.h. ohne Funktionseinschränkungen in Folge kardiovaskulärer Organschäden) zur Analyse herangezogen wurden. Die bei ihrer metaanalytischen Arbeit aufgetretenen Schwierigkeiten hinsichtlich uneinheitlicher Methodologie, Ein- und Ausschlusskriterien, Messintervalle u.v.m. fasste die Autorin später noch einmal zusammen [Yucha, 2002a] und löste mit ihren daraus resultierenden Empfehlungen für zukünftige Biofeedbackstudien kontroverse Diskussionen mit den beiden führenden Spezialisten für Blutdruckbiofeedback in den USA aus [Blanchard, 2002; McGrady, 2002], jedoch mit dem einenden Grundtenor der dringlichen Notwendigkeit weiterer Forschungsarbeit zum Blutdruckbiofeedback [Yucha, 2002b].

Grundsätzlich lassen sich zur Behandlung der Hypertonie verschiedene Biofeedbacktechniken einsetzen. Neben der direkten Rückmeldung des Blutdrucks (diastolisch oder systolisch) ist auch eine indirekte Beeinflussung des Blutdrucks über die Rückmeldung anderer Parameter möglich. Derartige indirekt auf den Blutdruck wirkende Methoden sind EMG-Feedback [z.B. Blanchard et al., 1993; Jurek al., 1992; McGrady al., 1991], Hauttemperatur-Feedback [z.B. Blanchard et al., 1996; Buby et al., 1990; McGrady, 1994; Latha und Kaliappan, 1991; Musso und Blanchard, 1991; Wittrock und Blanchard, 1992], respiratorisches Feedback [z.B. Franck et al., 1994a, b] oder Herzratenfeedback. Ihre Wirksamkeit beruht auf einer allgemeinen Reduzierung der sympathischen Aktivität. Zu diesen Biofeedbacktechniken wurde in den letzten 20-25 Jahren vermehrt Forschung betrieben, wohingegen die Forschungsaktivität zu direktem Blutdruckbiofeedback einen vergleichbar geringen Anteil ausmacht, auch weil noch bis vor Kurzem die technische Umsetzung einer noninvasiven, permanenten Messung Schwierigkeiten bereitete.

Lediglich Goebel et al. [1993], Hunyor et al., [1991, 1997] und Henderson et al. [1998] arbeiteten in jüngerer Zeit erfolgreich mit direktem Blutdruckfeedback. Unbefriedigend an den meisten hier zitierten Studien ist die Methode der Blutdruckmessung und -rückmeldung. Meist geschieht dies in technisch wenig befriedigender, halbautomatischer und überdies mehr oder weniger diskontinuierlicher Weise. Ein Transfer der experimentellen Methodologie auf den breiten klinischen Bereich ist in den meisten Fällen nicht denkbar. Es fehlt also immer noch an geeigneten Technologien, Methoden und praxisnahen Untersuchungen, die sich ausschließlich mit direktem, kontinuierlichem und vor allem hochfrequentem Blutdruckfeedback in Diagnostik und Therapie beschäftigen. Diese Mängel griff der Erstautor auf, was zur Entwicklung 
und der nachfolgend zu berichtenden Erprobung eines solchen Verfahrens führte.

\section{Patienten und Methoden}

Entwicklung eines Verfahrens für kontinuierliches Blutdruckfeedback Als unblutige und noninvasive Möglichkeit zur kontinuierlichen und phasengetreuen Messung des arteriellen Blutdrucks wurde das Prinzip der Gefäßwandentlastung von der Firma Ohmeda Medizintechnik GmbH (Berlin, Deutschland) zu dem hier verwendeten Gerät (2300 Finapres ${ }^{\mathrm{TM}}$ ) weiterentwickelt. Ein Finger des Probanden (in der Regel der Mittelfinger der nicht dominanten Hand) ist von einer aufblasbaren pneumatischen Manschette umgeben. Die Weite der Arterien unter der Manschette wird indirekt durch Messung der Transmission von infrarotem Streulicht erfasst und als Steuersignal für das Ventilsystem verwendet. Dieses steuert den Druck in der Manschette so, dass die Weite der Arterien konstant bleibt. Dazu muss der Manschettendruck phasengetreu dem Gefäßinnendruck nachgeführt werden, was bedeutet, dass die Drucke in den Fingerarterien und in der Manschette identisch sein müssen. Dieser Manschettendruck wird registriert und ist ein Äquivalent zum Blutdruck. Durch diese effiziente, aber auch aufwendige Kombination von Pneumatik und Elektronik wird eine phasengetreue «Beat-to-Beat»-Messung ermöglicht. Das Finapres-Gerät wurde dann über eine Verbindung der eingebauten Druckerschnittstelle mit der seriellen Schnittstelle eines handelsüblichen PCs in die Lage versetzt, die Messwerte digital zu speichern und rückzumelden (Abb. 1). Realisiert wurde dies durch eine selbst entwickelte Software über einen PC-Monitor, wo auf blauem Hintergrund die Darstellung eines der Form nach «altmodischen» schwarzen Riva-Rocci-Geräts mit Quecksilbersäule und beidseitig angeordneten weißen Ziffern zu sehen ist, dessen Säule seine Höhe analog zum diastolischen Wert ändert und deren Farbe gemäß der im Programmhintergrund errechneten Tendenz wechselt. Ein Belohnungssystem, in Form eines Kontostandfensters auf dem Bildschirm, verteilt Pluspunkte für eine fallende Tendenz und Minuspunkte für eine steigende Tendenz des diastolischen Drucks [Piesbergen et al., 1995, 1996].

Bei der therapeutischen Vorgehensweise orientierten wir uns hauptsächlich an den allgemeinen Prinzipien für Biofeedbackbehandlungen [Waschulewski-Floruß et al., 1994], wonach den Patienten Biofeedback als ein Verfahren vorgestellt wird, mit dem der Blutdruck sichtbar gemacht werden kann. Es soll auch aufgezeigt werden, dass mit dieser Methode eine individuell angewandte Strategie zur Blutdrucksenkung unmittelbar über- prüft werden kann. Nach einer halbstündigen Diagnostiksitzung wird in der Regel ein halbstrukturiertes Programm von 2-6 Sitzungen durchgeführt, wobei für eigene therapeutische Maßnahmen genügend Freiraum gelassen wird. Um diese Struktur zu verdeutlichen, werden nachstehend Instruktionen und Ablauf für die Therapie von hypertonen Patienten dargestellt:

\section{1. (Diagnostische) Sitzung}

(Nur bei unsicheren und aufgeregten Patienten, sonst gleich mit den Instruktionen der zweiten Sitzung beginnen.)

Der Patient nimmt auf dem Entspannungsstuhl Platz; gewöhnlichen Blutdruck erfragen, eventuell herkömmliche Manschettenmessung; hinweisen auf die Wichtigkeit einer eventuell bestehenden medikamentösen Therapie und deren weiterer Befolgung; Fragen nach Nikotin-, Alkohol- und anderer Genussgifteinnahme unmittelbar vor der Sitzung; nicht zu belehrend auftreten.

Manschette am 2. Glied des Mittelfingers der linken Hand anlegen, Finapres starten, Eichung abwarten. Computerprogramm starten, angestrebten Zielwert einstellen, Voreinstellungen übernehmen (keine Belohnung). Patienten instruieren hinsichtlich Bewegungsverhalten (zur Vermeidung von Artefakten); Bedeutung der wechselnden Farben erklären, reden und experimentieren lassen, auf den ständigen Druckwechsel vor allem, wenn der Patient spricht, hinweisen. Die Patienten machen meist schon in der diagnostischen Sitzung die für sie überraschende Erfahrung, dass sie Möglichkeiten haben, ihren Blutdruck selbst zu beeinflussen. Am Ende der Sitzung den aktuellen Kontostand notieren, die Auswertung vornehmen lassen und auf Wunsch mit dem Patienten besprechen.

\section{Sitzung}

Anfang wie oben, jedoch geeignete Belohnungsmodalität einstellen und erklären.

Entspannende Atmosphäre herstellen, den Patienten zur Eigenentspannung ermutigen, die Wahrnehmung auch auf den Atemvorgang richten, die Phase des Ausatmens verlängern lassen, auf den meist drucksenkenden Effekt mittels der Bildschirmdarstellung hinweisen und unterstützen. Bei Unfähigkeit zur Eigenentspannung therapeutisch eingreifen, jedoch nicht mit Progressiver Muskelrelaxation (wegen Gefahr von Bewegungsartefakten), sondern eher mit mental orientierten Verfahren (z.B. Autogenes Training). Ende wie oben.

\section{3.-4. Sitzung}

Ablauf wie in 2. Sitzung; bei guter Entspannungsfähigkeit des Patienten den Raum nach Rücksprache verlassen und nur zwischendurch die Werte
Abb. 1. Schema der Feedbackschleife.

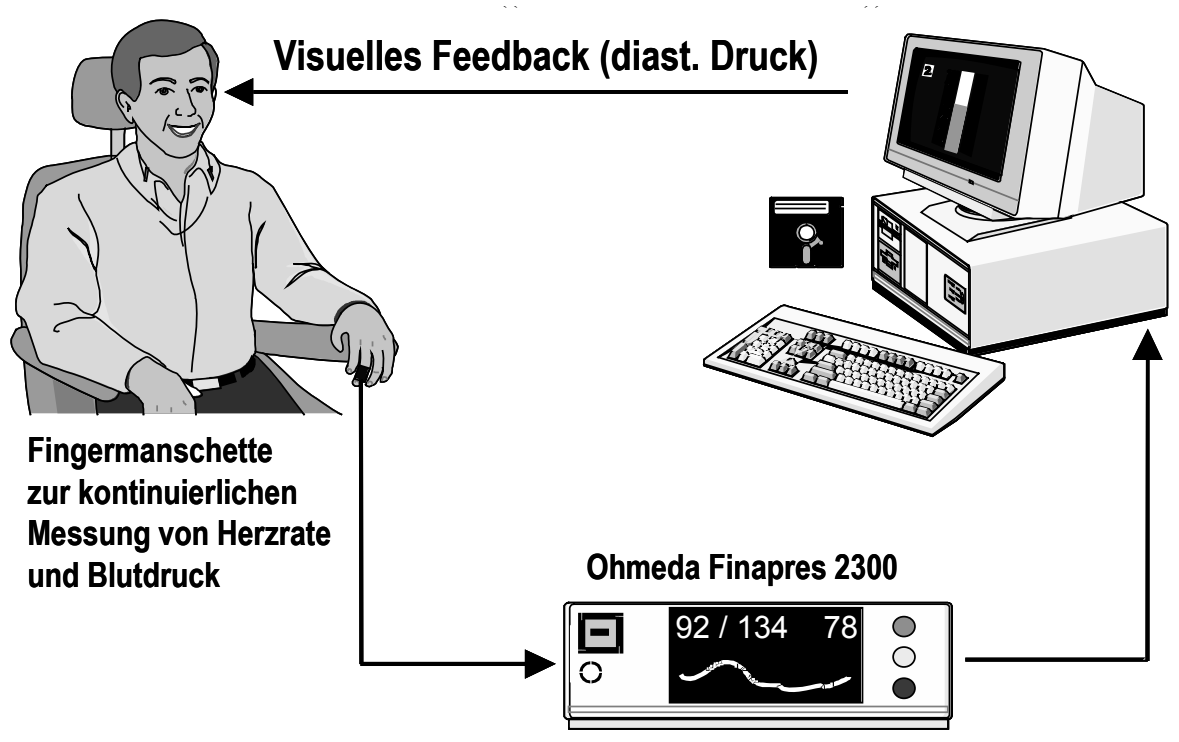


Tab. 1. Stichprobencharakteristika der Versuchsgruppen

\begin{tabular}{llllllll}
\hline Gruppe & $\begin{array}{l}\text { Aufenthaltstage } \\
(\mathrm{M} \pm \mathrm{SD})\end{array}$ & $\begin{array}{l}\text { Alter } \\
(\mathrm{M} \pm \mathrm{SD})\end{array}$ & $\begin{array}{l}\text { Aufnahme } \\
\text { gewicht, kg } \\
(\mathrm{M} \pm \mathrm{SD})\end{array}$ & $\begin{array}{l}\text { Sitzungen } \\
(\mathrm{n})\end{array}$ & $\begin{array}{l}\text { Männer } \\
(\mathrm{n})\end{array}$ & $\begin{array}{l}\text { Frauen } \\
(\mathrm{n})\end{array}$ & $\begin{array}{l}\text { Gesamt } \\
(\mathrm{n})\end{array}$ \\
\hline $\mathrm{K} 1$ & $30,50 \pm 5,55$ & $52,55 \pm 10,24$ & $86,82 \pm 13,76$ & 0 & 14 & 8 & 22 \\
$\mathrm{~K} 2$ & $30,10 \pm 5,13$ & $53,28 \pm 8,09$ & $88,75 \pm 15,67$ & 2 & 13 & 7 & 20 \\
$\mathrm{E} 1$ & $30,80 \pm 5,75$ & $52,30 \pm 6,93$ & $84,40 \pm 13,30$ & 2 & 15 & 5 & 20 \\
$\mathrm{E} 2$ & $36,27 \pm 6,63$ & $53,36 \pm 9,58$ & $89,45 \pm 18,82$ & 3 & 4 & 3 & 7 \\
& & & 4 & 4 & 0 & 4 \\
& & & 5 & 5 & 3 & 8 \\
\hline
\end{tabular}

am Finapres kontrollieren (leise zur Tür hereinsehen; besser: Videoüberwachung oder Einwegscheibe). Ende wie oben.

\section{5.-6. Sitzung}

«Konfrontationssitzungen», nur auf ausdrücklichen Wunsch und mit Einverständnis des Patienten.

Therapeut und Patient können zusammen am Bildschirm beobachten, wie sich der Blutdruck ändert, wenn bestimmte Situationen besprochen, vorgestellt oder bearbeitet werden. Hierbei wird das Hauptgewicht auf eine Art Einsichtstherapie gelegt, die neben der Entspannung die Sensibilität für den eigenen Körper, seine Funktionen und Signale verbessern soll, um aufgrund der damit verbundenen Prozesse auch ungesunder Lebensweise und Risikofaktoren wie Rauchen, Alkohol und falscher Ernährung entgegenzuwirken. Ende wie oben.

\section{Studiendesign und Versuchsablauf}

Zur anschließenden Evaluation wurde das Verfahren auf zwei Stationen der Klinik Höhenried in Bernried bei München als Erweiterung des normalen Klinikprogramms aufgenommen. Da das normale Klinikprogramm weitgehend standardisiert abläuft, damit kein Patient begünstigt oder benachteiligt wird, kann davon ausgegangen werden, dass unterschiedliche Effekte von der neuen zusätzlichen Behandlung herrühren müssen. $\mathrm{Zu}$ deren Evaluation wurde ein quasiexperimentelles Kontrollgruppendesign mit zwei Experimentalgruppen (E1 und E2) und zwei «historischen» Kontrollgruppen (K1 und K2) mit Daten aus früheren Kohorten verwendet. Eine Randomisierung auf Kontroll- und Experimentalgruppen war daher nicht möglich, nur eine Parallelisierung oder die Bildung von «Matched Samples» [z.B. Bortz und Döring, 1995]. Da man somit bei unseren Vergleichsgruppen nicht vorbehaltlos von «echten» Kontrollgruppen ohne jegliches Treatment sprechen kann, wird der Begriff der «Kontrollgruppe» bisher und fortan im Sinne einer Vergleichsgruppe verwendet.

In den Versuchsbedingungen saßen die Patienten in einem ärztlichen Untersuchungszimmer der Klinik vor dem PC-Monitor auf einem Entspannungsstuhl. In der Regel - außer bei Handverletzungen - wurde die Fingermanschette des Finapres-Geräts am 2. Glied des Mittelfingers der nicht dominanten Hand angelegt (Abb. 1). Als Versuchsleiterin $\mathrm{sa} ß$ die Zweitautorin während jeder der 2-6 halbstündigen Biofeedbackbehandlungen in einem Stuhl ungefähr $2 \mathrm{~m}$ links neben dem Probanden. Der Ablauf der Biofeedbacksitzungen orientierte sich im Wesentlichen an der oben aufgeführten Vorgehensweise, wobei aber schon bei der 1. Sitzung mit den Instruktionen zur 2. Sitzung begonnen wurde. Die 5. und 6. Sitzung waren «Konfrontationssitzungen», in denen versucht wurde, die Patienten mithilfe der Bildschirmanzeige an eventuelle psychische Ursachen ihres Herzleidens heranzuführen. Die zweite Kontrollgruppe (Pseudofeedback) instruierte eine andere Versuchsleiterin.

Im Standardprogramm der Klinik, das alle Probanden durchliefen, ist die Grundlage für die psychotherapeutische Betreuung ein integratives Modell, wonach 2-mal wöchentlich für alle Patienten Gesprächsgruppen zur psychischen Verarbeitung und Bewältigung ihrer Erkrankung stattfinden. Weitere Maßnahmen der Betreuung sind fortlaufende und geschlossene themenzentrierte Gruppen sowie Einzeltherapien. Zur Gesundheitsinformation über Nutzen und Gefahren der körperlichen Belastung bei Herzkranken findet wöchentlich eine Seminarstunde statt. Ein weiterer wichtiger Punkt der therapeutischen Betreuung besteht in fortlaufenden oder geschlossenen Entspannungsgruppen. Die Entspannungsübungen orientieren sich an der Progressiven Muskelrelaxation nach Jacobson [Ohm, 1992]. Da viele Patienten zusätzlich zu Herz-Kreislauf-Erkrankungen an Adipositas, Diabetes mellitus oder multiplen Stoffwechselstörungen leiden, besteht die Möglichkeit zu vielfältigen Gruppen- oder Einzelberatungen im Bereich Ernährung und Diätetik, um die mitunter unzureichenden Ess- und Trinkgewohnheiten zu reflektieren und nach der Heilbehandlung im häuslich-beruflichen Alltag ein gesünderes Ernährungsverhalten anzustreben.

\section{Stichprobe}

Die Probanden wurden uns von den Teams zweier Stationen der Klinik zugewiesen und waren alle diagnostizierte Hypertoniker. Die Stationsteams, denen auch der Drittautor angehörte, hatten keinerlei Einfluss darauf, welche Patienten gerade von ihnen stationär behandelt wurden, denn diese wurden von verschiedensten Institutionen und Ärzten deutschlandweit zur Anschlussheilbehandlung dorthin überwiesen. Dies spricht jedoch für die Repräsentativität der Stichprobe. Die Entscheidung über die Teilnahme eines Patienten am Biofeedbacktraining trafen Stationsärzte, Psychologen und Pflegepersonal der betreffenden Stationen gemeinsam. Die Stichprobengröße wurde durch die organisatorischen Gegebenheiten der Klinik bestimmt, weswegen a priori keine Berechnungen zum erforderlichen Stichprobenumfang durchgeführt wurden. Somit erhielten wir 84 Probanden, von denen je 42 auf die Kontrollgruppen und auf die Experimentalgruppen entfielen. Tabelle 1 zeigt die Verteilung der Probanden sowie weitere Stichprobenkennwerte.

Um aus vielen verschiedenen Wirkfaktoren den durch Biofeedback entstandenen Effekt isolieren zu können, versuchten wir in den Experimentalgruppen Stichprobencharakteristika, von denen ein Einfluss auf die Entwicklung des Blutdrucks während des Klinikaufenthaltes anzunehmen war, vergleichbar zu halten. Derartige intervenierende Variablen sind neben der Aufenthaltsdauer (Länge der Erholungszeit) zusätzlich erhaltenes respiratorisches Feedback (Reduzierung der allgemeinen sympathischen Aktivität) sowie Medikamenteneinnahme (An-, Ab- oder Umsetzen der Medikation). Zur Kontrolle dieser Variablen wurde ein MatchingVerfahren eingesetzt. Weitere Behandlungselemente wurden hier nicht berücksichtigt, da alle Teilnehmer der Experimental- und der Kontrollgruppen eine nahezu in allen Punkten des Klinikprogramms identische Behandlung durchliefen. Einen weiteren wichtigen Aspekt der Vergleichbarkeit der Versuchsgruppen stellte die Verteilung der Krankheitsdiagnosen dar. Auch hier konnte in einigen Diagnosen bedingt Vergleichbarkeit erreicht werden (Tab. 2). 
Tab. 2. Verteilung der Krankheitsdiagnosen (Angaben in \%)

\begin{tabular}{lcccc}
\hline & E1 (n=20) & E2 (n=22) & K1 (n=22) & K2 (n=20) \\
\hline Arterielle Hypertonie, Hypertonus & 100,0 & 100,0 & 100,0 & 100,0 \\
Embolien, Thrombosen & 5,0 & 13,64 & 4,55 & 10,0 \\
Herzgefäßerkrank., Klappenfehler & 10,0 & 13,64 & 27,27 & 15,0 \\
Andere Herzerkrankungen & 10,0 & 4,55 & 13,64 & 10,0 \\
Bluterkrankungen & 5,0 & 4,55 & 18,18 & 10,0 \\
Nierenerkrankungen & 5,0 & 0,0 & 13,64 & 5,0 \\
Periphere Gefäßerkrankungen & 20,0 & 22,73 & 50,0 & 25,0 \\
Herzrhythmusstörungen & 15,0 & 18,18 & 36,36 & 20,0 \\
Obstruktive Lungenerkrankungen & 15,0 & 4,55 & 13,64 & 10,0 \\
Stress - psychische Belastung & 40,0 & 68,18 & 22,73 & 45,0 \\
\hline
\end{tabular}

Ebenfalls unter Hypertonie sowie deren Folgeerkrankungen litten die Probanden der Kontrollgruppen. Sie stammten aus den gleichen Stationen wie die Teilnehmer der Experimentalgruppen und waren ein halbes Jahr (K1), bzw. unmittelbar vor Einführung des Blutduckbiofeedback (K2) zur Anschlussheilbehandlung in Höhenried gewesen. Dies erwies sich als notwendig, um gleiches Treatment durch dieselben Therapeuten zu gewährleisten und um zu vermeiden, dass sich Probanden von der Teilnahme am Biofeedback ausgeschlossen fühlen könnten. Die Kontrollbedingung hatte zwei Ausprägungen: die Gruppe K1 erhielt nur das Standardprogramm der Klinik; die Gruppe K2 bestand aus Patienten, die zusätzlich 2 halbstündige Sitzungen mit dem Finapres-Gerät erhielten. Allerdings handelte es sich dabei nicht um unser softwaregesteuertes Blutdruckfeedback, sondern nur um die Anweisung, die Anzeigen im kleinen eingebauten Monitor des Finapres-Geräts zu beobachten und damit zu versuchen, willentlich den Blutdruck zu senken. Die Instruktion entsprach somit im Wesentlichen der für die erste diagnostische Sitzung, bis auf die technisch bedingten Unterschiede. Der Monitor zeigt neben einer komprimierten Verlaufskurve von diastolischem und systolischem Druck auch deren Werte und die der Herzrate digital an (Abb. 1), so dass auch hier eine eingeschränkte Art von Feedback erfolgen kann. Diese Bedingung kann Aufschluss darüber geben, ob nicht allein schon das Angeschlossensein an das Gerät und die Beobachtung und bloße Rückmeldung der Werte ohne Verstärkung von positiven Konsequenzen zu einem blutdrucksenkenden Effekt führen kann. Auf diese Weise wurde eine partielle Kontrolle des Placeboeffekts möglich.

\section{Fragestellung und Auswertung}

Als Fragestellung zu den Effekten der zusätzlichen Biofeedbackbehandlung im Klinikalltag wurden vier Hypothesen gebildet:

1. Die Anwendung von direktem Blutdruckbiofeedback führt bei den Probanden der Experimentalgruppen zu höheren Blutdrucksenkungen im Klinikalltag als bei jenen der Kontrollgruppen.

2. Probanden, die am Finapres-Gerät ohne Biofeedback übten, erzielen höhere Blutdrucksenkungen im Klinikalltag als Patienten, die sich nur der Klinikbehandlung unterzogen.

3. Probanden mit 2 Biofeedbacksitzungen erzielen höhere Blutdrucksenkungen im Klinikalltag als Patienten, die am Finapres-Gerät ohne Biofeedback übten.

4. Probanden mit 3 oder mehr Biofeedbacksitzungen erzielen höhere Blutdrucksenkungen im Klinikalltag als Patienten, die nur 3-mal am Biofeedback teilnahmen.

Für die Beurteilung eines Behandlungseffekts im Sinne von Blutdrucksenkungen wurde als abhängige Variable (AV) die Differenz der Mittelwerte aus den meist täglich gemessenen Blutdruckwerten der ersten und der Entlassungswoche gebildet. Jeder Proband musste mindestens 3 Blutdruckmessungen in der ersten und letzten Woche aufweisen. Der Blutdruck wurde einmal täglich, wenn notwendig auch mehrmals am Tag während der gesamten Heilbehandlung vom Klinikpersonal nach der Riva-
Rocci-Methode mittels Armmanschette gemessen. Zur Mittelung und Differenzbildung wurde der medizinische Mitteldruck (MAP) herangezogen, berechnet aus dem systolischen und diastolischen Blutdruck nach der Formel: MAP $=\mathrm{p}_{\text {dias }}+0,333 *\left(\mathrm{p}_{\mathrm{sys}}-\mathrm{p}_{\text {dias }}\right)$. Diese Differenzvariable wurde einem Kolmogorov-Smirnov- und einem Levine-Test unterzogen und erwies sich als normalverteilt $(\mathrm{Z}=0,733 ; \mathrm{p}=0,656)$ und varianzhomogen $(\mathrm{F}=2,683 ; \mathrm{p}=0,052)$.

Für die Mittelwertvergleiche des Gruppenfaktors (UV) kamen univariate, 1-faktorielle Kovarianzanalysen der Differenzwerte des MAP (AV) nach dem allgemeinen linearen Modell zur Anwendung; daran anschließend wurden zur Untersuchung signifikanter Effekte zwischen den Gruppen noch A-priori-Vergleiche im Sinne von wiederholten Kontrasten mit dem Programmsystem SPSS 14.0 gerechnet. Als Signifikanzniveau für alle statistischen Verfahren wurde ein $\alpha$-Risiko von $5 \%$ gewählt.

\section{Ergebnisse}

Im Vergleich der Blutdruckmittelwerte vor und nach der Heilbehandlung zeigte sich, dass beide Experimentalgruppen, im gewogenen Mittel zusammengenommen, diastolisch / systolisch eine Senkung von 7,48 / 12,15 mm Hg erreichten, bei Standardabweichungen (SD) von 10,47 / 16,37 mm Hg, während es die Kontrollgruppen im Mittel nur auf eine Senkung von 2,87 / 2,56 mm Hg ( $\mathrm{SD}=6,66$ / 10,35 mm Hg) brachten. Abbildung 2 zeigt deutlich den Effekt der Biofeedbackbehandlung im Sinne höherer Senkungen für die Experimentalgruppen. Ebenfalls zu erkennen ist, dass sich die Differenzmittelwerte bei beiden Kontrollgruppen weder in der ersten noch in der letzten Woche besonders auffällig unterscheiden. Die Abbildung zeigt darüber hinaus das höhere Ausgangsniveau für die E2 zu Beginn der Heilbehandlung.

Damit sichergestellt werden konnte, dass die verschiedenen Ausgangsniveaus der Gruppen keine Verzerrung der Resultate (z.B. Regression zur Mitte) bewirken, wurde nach einer Empfehlung von Vickers und Altman [2001] der Mittelwert des MAP in der ersten Woche (Baseline) als Kovariate mit einbezogen. Weiterhin fanden wir einen signifikanten $\mathrm{Zu}$ sammenhang zwischen der Anzahl erhaltener Biofeedbacksitzungen und der Aufenthaltsdauer in der Klinik ( $\mathrm{r}=0,357$; $\mathrm{p}<$ 0,001). Dies bedeutet insbesondere für E2 eine potentielle Erhöhung der Effekte allein schon durch den längeren Aufenthalt, weswegen auch diese Störvariable in die Analyse einge- 
Abb. 2. Differenzmittelwerte des Blutdrucks (prä/post) in mmHG.

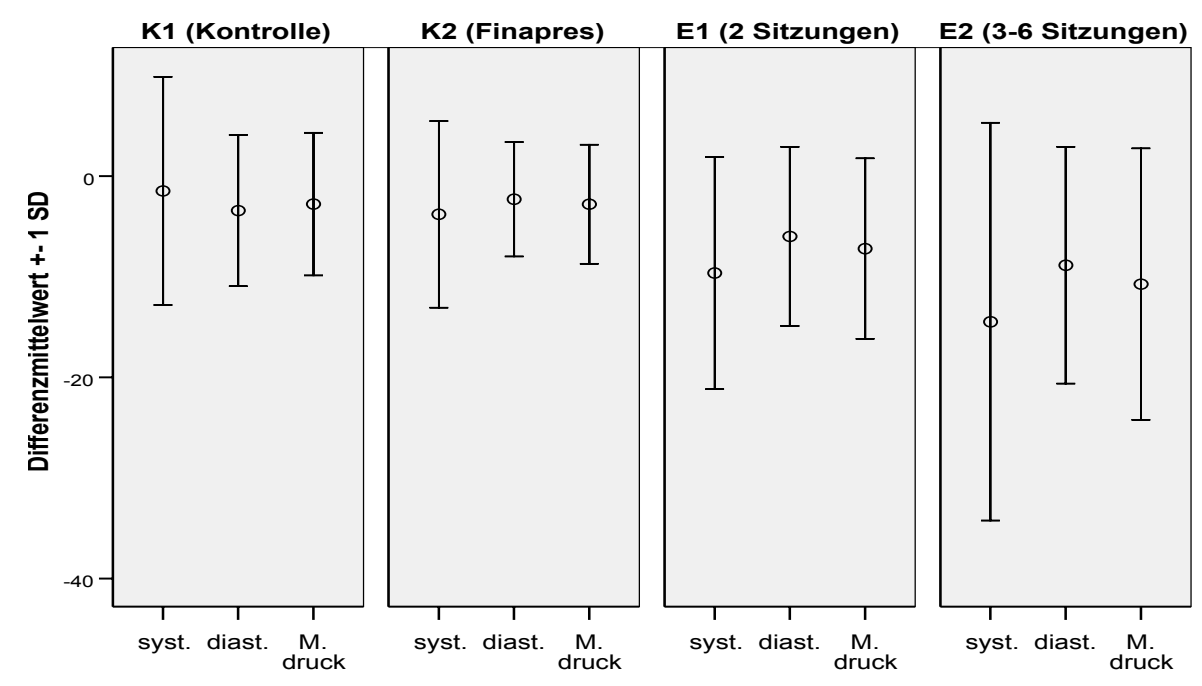

bunden und somit kontrolliert wurde. Die anschließend durchgeführte Kovarianzanalyse ergab für die AV «Differenz des MAP» eine Signifikanz auf dem 5\%-Niveau sowohl beim Gruppenfaktor (Ausprägungen Experimental/Kontrolle: $\mathrm{F}=11,721 ; \mathrm{df}=1 ; \mathrm{p}=0,001)$, wie auch beim Baseline-MAP $(\mathrm{F}=68,403 ; \mathrm{df}=1 ; \mathrm{p}<0,001)$, was die Einbeziehung der unterschiedlichen Ausgangsniveaus rechtfertigt. Nicht signifikant wurde die Aufenthaltsdauer $(\mathrm{F}=0,000 ; \mathrm{df}=1 ; \mathrm{p}=0,983)$, was auf ihren geringen Einfluss als Störvariable hinweist.

Im Rahmen einer zweiten Analyse über alle vier Gruppen und mit denselben Kovariaten ermöglichten wiederholte Kontraste einen Vergleich benachbarter Kategorien, wobei auf eine Korrektur des Signifikanzniveaus verzichtet werden konnte, da es sich um a priori spezifizierte Einzelhypothesen handelte [Bortz et al., 2000, S. 53f]. Wie in Tabelle 3 zu sehen, ergaben sich im ersten Kontrast der zusammengenommenen Kontroll- und Experimentalgruppen und im dritten Kontrast Signifikanzen, im zweiten und vierten Kontrast zwischen K1 und K2 bzw. E1 und E2 jedoch keine signifikanten Unterschiede.

Die Annahme von Hypothese 1 kann nun als inferenzstatistischer Beleg für die Wirksamkeit von zusätzlichen Behandlungen mit dem kontinuierlichen Blutdruckfeedback gelten. Das bloße Üben mit dem Finapres-Gerät zusätzlich zum Kliniktreatment hat offenbar keinen nennenswerten Einfluss auf die Höhe des Blutdrucks, somit muss für Hypothese 2 die Nullhypothese beibehalten werden. Demgegenüber scheint aber nach Annahme von Hypothese 3 eine nur 2-malige Feedbackbehandlung eine bessere Wirkung und auch bereits ausreichenden Effekt zu haben, da sich die Gruppe mit 3 oder mehr Sitzungen zwar in den Stichprobenmittelwerten, aber dennoch nicht signifikant von der nur 2-mal behandelten Gruppe unterscheidet, was für Hypothese 4 die Beibehaltung der Nullhypothesee bedeutet.

Eine weitere Möglichkeit zur Erhärtung der Wirksamkeit einer Behandlungsmethode ist die Berechnung von Effekt- stärken. Zur Ermittlung der individuellen Effektstärken wurde bei jedem Probanden der Wert des MAP in der letzten Woche von dem in der ersten Woche subtrahiert und das Ergebnis durch die Standardabweichung der Gesamtgruppe dividiert. Im vorliegenden Fall läuft dieses Vorgehen letztendlich auf eine Standardisierung der Druckdifferenzen des MAP hinaus. Im Ergebnis wird ersichtlich, dass die mittleren Effektstärken bei K1 $(0,2805)$ und K2 $(0,2821)$ nahezu gleich sind, jedoch bei E1 $(0,7308)$ und E2 $(1,0893)$ wesentlich höher liegen. Nach Cohen [1988] hätte die Klinikbehandlung insgesamt mit 0,5999 eine moderate, in Verbindung mit unserer Biofeedbackbehandlung eine hohe $(0,9186)$ und ohne diese eine geringe $(0,2812)$ mittlere Effektstärke.

Als zusätzliches Kriterium wurden auch noch die Anzahlen der Therapie-Responder aus den einzelnen Gruppen ermittelt. Somit befinden sich allein in E1 fast doppelt so viele Responder (13 Probanden) wie in beiden Kontrollgruppen zusammen (4 Probanden) und in der Gruppe mit 3-6 Sitzungen genau 3-mal so viele Responder (12 Probanden: $\chi^{2}=17,226$; $\mathrm{df}=3 ; \mathrm{p}=0,001)$. In der Relation sinkt die Zahl der Non-Responder von 38 Probanden in den Kontrollgruppen auf 13 (E1) und auf 10 (E2). Das Responder-Kriterium lag hierzu bei einer Druckdifferenz von mindestens $10 \mathrm{~mm} \mathrm{Hg}$ systolisch und $5 \mathrm{mmHg}$ diastolisch zwischen erster Woche und Entlassungswoche. Wir orientierten uns hierbei an den Vorschlägen von Franck et al. [1994b].

\section{Diskussion}

Das Verfahren des kontinuierlichen Blutdruckfeedback wurde bereits an verschiedenen Einrichtungen (z.B. Klinik Höhenried, Reha-Klinik Spreewald, psychosomatische Klinik Roseneck am Chiemsee [Marwitz, 2000], Kurklinik Kneippianum in Bad Wörishofen, Reha-Klinik Wiessee) in das Therapieprogramm integriert. Deshalb entschieden wir uns bei der 
Tab. 3. Kontrast-Tests für die Druckdifferenzen

\begin{tabular}{lllll}
\hline & Kontrast & $\begin{array}{l}\text { Kontrast- } \\
\text { schätzer }\end{array}$ & $\begin{array}{l}\text { Standard- } \\
\text { fehler }\end{array}$ & $\mathrm{p}$ \\
\hline Druckdifferenz & $1(\mathrm{~K}-\mathrm{E})$ & 5,412 & 1,581 & 0,001 \\
(Mitteldruck) & 2(K1 - K2) & 0,568 & 2,174 & 0,794 \\
& 3(K2-E1) & 5,026 & 2,227 & 0,027 \\
& $4(\mathrm{E} 1-\mathrm{E} 2)$ & 1,506 & 2,311 & 0,517 \\
\hline
\end{tabular}

Evaluation von vornherein, mit Felduntersuchungen zu arbeiten im Sinne hoher ökologischer Validität [Fahrenberg et al., 2002], also unter realitätsnahen und gleichzeitig auch kontrollierten Bedingungen, möglichst im Rahmen einer größeren Institution mit hohem Patientenaufkommen. In der Klinik Höhenried konnte trotz der verschiedenen Ausgangsniveaus einiger Patienten vor der Behandlung belegt werden, dass die zusätzliche Behandlung mit kontinuierlichem Blutdruckbiofeedback eine größere Senkung des Blutdrucks bewirkte als das Kliniktreatment allein oder nur das zusätzliche Üben mit dem Finapres-Gerät, was einer Art Placebobedingung gleichgestellt werden kann. Dies steht im Gegensatz zu den Erkenntnissen von Grawe et al. [1994], die in ihrer Metastudie nur dann eine signifikante Wirkung für Biofeedbacktreatments fanden, wenn sie Kontrollbedingungen gegenübergestellt wurden, in denen die Patienten wirklich keinerlei Behandlung erhalten hatten. Ferner hat sich gezeigt, dass es offenbar keinen statistisch bedeutsamen Unterschied macht, ob man lediglich mit 2 Biofeedbacksitzungen oder mit 3 und mehr Behandlungen arbeitet, obwohl gerade die Gruppe mit 3-6 Sitzungen allein durch ihr höheres Ausgangsniveau a priori mehr Chancen auf höhere Senkungsbeträge gehabt hätte [Vaitl, 1994]. Das könnte bedeuten, dass der schon nach kurzer Zeit (2 Sitzungen) eintretende Effekt durch Übung zwar verbessert wird, wohl aber nicht in einem Ausmaß, das den dafür zu betreibenden Aufwand rechtfertigt.

Es scheinen im Wesentlichen zwei Komponenten für den erzielten Effekt verantwortlich zu sein: eine schneller wirksame, kognitive Komponente, die so etwas wie das Konzept der erlebten Selbstwirksamkeit [Bandura, 1977] und der Selbstkontrolle [Meichenbaum, 1976] darstellt, und die etwas langsamere Komponente der Übung, die wohl eher einen allgemeinen Entspannungseffekt bewirkt. Auch Blanchard [1990] stellte fest, dass die Selbsteffizienzerwartung und in der Folge die Motivation erhöht werden, wenn die erfolgreiche Beeinflussung eines Parameters rückgemeldet wird. Rief et al. [1996] sehen die Erhöhung der Selbstwirksamkeitserwartung sogar als eines der obersten Ziele. Nach diesen Erkenntnissen könnte die Dauer von hochfrequenten Biofeedbackbehandlungen verkürzt werden, was wegen geringerem Zeit- und Kostenaufwand für Patienten, Therapeuten und Kostenträger gleichermaßen interessant sein dürfte.

Weiterhin erfolgte eine Unterteilung der Gesamtstichprobe in Responder und Non-Responder, da nach McGrady und Hig- gins [1989] davon auszugehen war, dass nicht alle Probanden in gleicher Weise vom Biofeedback profitieren. Sie fanden als Hauptmerkmal für die Responder in ihrer Stichprobe Faktoren, die sich mit dem Begriff «neurovegetative Hyperreagibilität» umschreiben lassen. Franck et al. [1994b] hatten eine 5-Cluster-Lösung für die Probanden ihrer Studie gefunden, die sich an mittelfristig überdauernden Effekten orientierte. Sie konnten die in Blutdruck- und Herzratensenkung erfolgreichsten Versuchspersonen durch psychologische Charakteristika beschreiben wie Interesse an Spiel und Technik, ein hohes Maß an aktiver Stressbewältigung und keine Persönlichkeitsauffälligkeiten. McGrady [1996] vertritt darüber hinaus die Ansicht, dass Personen mit einem hyperreagiblen Vegetativum (hohe Herzrate, kalte Hände, hohe Muskelspannung, hohe Plasma-Renin-Aktivität) als gute BiofeedbackResponder gelten können. Dies dürfte sicherlich sowohl den Interessen der Patienten entgegenkommen, die dann eine optimal auf sie zugeschnittene Therapie erhalten könnten, wie auch aus ökonomischen Gründen denen der Kostenträger.

Aufgrund der Ergebnisse in Höhenried soll nun das direkte Blutdruckfeedback nicht als wiederentdecktes Wundermittel gegen Hypertonie angepriesen werden. Vielmehr wird betont, dass hiermit nur eine zusätzliche Variante im Reigen der Hochdruckbehandlung ihre ersten Prüfstandläufe absolviert hat. Beispiele integrativen Vorgehens sind das Konzept der Klinik Höhenried und der Klinik Roseneck in Prien am Chiemsee [Marwitz, 2000], die ebenfalls mit unserem Verfahren arbeitet. Eine Möglichkeit zur Außerachtlassung bekannter Risikofaktoren auf der somatischen, psychischen und der Verhaltensebene sowie einen vollständigen Ersatz der pharmakologischen Standardintervention will und kann auch dieses Verfahrensprinzip nicht bieten. Schädliche Nebenwirkungen oder Gegenanzeigen sind bis jetzt noch nicht beobachtet worden. Aber zum Beispiel bei akuten Hochdruckkrisen, wo es um Leben oder Tod gehen kann, greifen Medikamente schneller und auch sicherer als Biofeedback. Zudem waren fast alle Patienten unserer Studie mediziert, was sowieso bedeutet, dass das Biofeedbackverfahren nicht eine Alternative zur Pharmakotherapie bilden soll, sondern hauptsächlich als adjuvante Maßnahme einer medizinischen Behandlung eingesetzt werden soll, wie dies auch schon Vaitl [1982b] vorschlägt.

Weitere Limitierungen unserer Studie liegen vor allem in der fehlenden Randomisierung bei der Gruppenzuteilung. Auch konnte durch unsere Studie nicht geklärt werden, wie lange die während der Heilbehandlung mittelfristig erzielten Drucksenkungen über die Dauer des Klinikaufenthalts hinaus wirksam sind. Durch die Rückkehr der Patienten aus der «geschützten» Klinikumgebung in das jeweilige soziale Umfeld könnten aufgrund von Rückfällen in schlechte Ernährungsgewohnheiten, Bewegungsmangel, Stress im Beruf etc. wieder Blutdruckerhöhungen eintreten. Sodann wirken sich die unterschiedlichen Zeitpunkte und Abstände der Biofeedbacksitzungen einschränkend auf die Reliabilität aus. Ferner sollten noch die vom Aufzeichnungsprogramm innerhalb der Sit- 
zungen abgespeicherten Daten analysiert, interpretiert und gegebenenfalls publiziert werden. Nachteilig sind auch die Gebundenheit an einen stationären Einsatz sowie die Beschaffungsschwierigkeiten des Finapres-Geräts zur Blutdruckmessung, da es nicht mehr vertrieben wird. Diese Probleme sind aber nicht psychologischer, sondern rein ökonomischer und technologischer Natur. Alternativen stellen das Portapres $2.0^{\circledR}$-Gerät von TNO-BMI ${ }^{\mathrm{TM}}$ [Fahrenberg et al., 1997] sowie auch die Colin ${ }^{\mathrm{TM}}$ BP 508-T ${ }^{\circledR}$-Anlage [Zorn et al., 1997] dar.
Beide Geräte liefern aber keine zuverlässigen Absolutwerte des Blutdrucks, sondern nur Relationen von Unterschieden. Das Portapres-Gerät hat den Vorteil, dass es transportabel am Probanden angebracht werden kann und somit beschränkt auch für längere Monitorings in wechselnder Umgebung geeignet ist. Aus heutiger Erfahrung zur rasanten Entwicklung der Mikroelektronik dürfte es sich nur noch um wenige Jahre handeln, bis vergleichbare Anlagen auf Taschenformat geschrumpft sind.

\section{Literatur}

Andrews G, Macmahon SW, Austin A, Byrne DG: Comparison of drug and non-drug treatments. BMJ 1982;284:1523-1526.

Bandura A: Self-efficacy: Toward a unifying theory of behavioural change. Psychol Rev1977;84:191-215.

Birbaumer N, Schmidt RF: Biologische Psychologie. Berlin, Springer, 1990.

Blanchard EB: Biofeedback treatments of essentia hypertension. Biofeedback Selfregulation 1990;15:209228.

Blanchard EB: Biofeedback and hypertension: A déjà vu experience - Comments on Yucha's 'Problems inherent in assessing biofeedback efficacy studies'. Appl Psychol Biofeedback 2002;27(1):107-109.

Blanchard EB, Eisele G, Gordon MA, Cornish PJ, Wittrock DA, Gilmore L, Vollmer AJ, Wan C: Thermal biofeedback as an effective substitute for sympatholytic medication in moderate hypertension: a failure to replicate. Biofeedback Selfregulation 1993;18(4):237253.

Blanchard EB, Eisele G, Vollmer A, Payne A, Gordon M, Cornish P, Gilmore L: Controlled evaluation of thermal biofeedback in treatment of elevated blood pressure in unmedicated mild hypertension. Biofeedback Selfregulation 1996;21(2):167-190.

Bortz J, Döring N: Forschungsmethoden und Evaluation. Berlin, Springer, 1995.

Bortz J, Lienert GA, Boenke K: Verteilungsfreie Methoden in der Biostatistik. Berlin, Springer, 2000.

Buby C, Elfner LF, May IG: Relaxation pretraining, pulse wave velocity and thermal biofeedback in the treatment of essential hypertension. Int J Psychophysiol 1990;8(3):225-230.

Cohen J: Statistical Power Analysis for the Behavioral Sciences. Hillsdale, NY, Lawrence Erlbaum, 1988.

Fahrenberg J, Foerster F, Müller W: Non-invasive estimations of ventricular ejection time and stroke volume: comparison of impedance cardiography and the Portapres 2. J Med Eng Technol 1997;21(1):15-22.

Fahrenberg J, Leonhart R, Foerster F: Alltagsnahe Psychologie. Bern, Huber, 2002.

Franck M, Schäfer H, Stiels W, Wassermann R, Hermann JM: Entspannungstherapie mit dem respiratorischen Feedback bei Patienten mit essentieller Hypertonie. Psychother Psychosom Med Psychol 1994a;44(910):316-322.

Franck M, Schäfer H, Stiels W, Wassermann R, Hermann JM: Welche Patienten profitieren von einer Behandlung mit dem respiratorischen Feedback (RFB)? Psychother Psychosom Med Psychol 1994b;44(11): 390-395.

Goebel M, Viol GW, Ovebaugh C: An incremental model to isolate specific effects of behavioral treatments in essential hypertension. Biofeedback Selfregulation 1993;18(4):255-280.
Grawe K, Donati R, Bernauer F: Von der Konfession zur Profession. Göttingen, Hogrefe, 1994. Henderson RJ, Hart MG, Lal SKL, Hunyor SN: The effect of home training with direct blood pressure biofeedback of hypertensives: a placebo-controlled study. J Hypertension 1998;16(6):771-778.

Hunyor SN, Bartrop R, Craig A, Cejnar M, Liggins G, Henderson RJ, Jones M: Voluntary blood pressure control by biofeedback: relationship to psychological characteristics. Clin Exp Pharmacol Physiol 1991;18(1):5559.

Hunyor SN, Henderson RJ, Lal SKL, Carter NL, Kobler H, Jones M, Bartrop RW, Craig A, Mihailidou AS: Placebo-controlled biofeedback blood pressure effect in hypertensive humans. Hypertension 1997;29:12251231.

Jurek IE, Higgins JT, McGrady A: Interaction of biofeedback-assisted relaxation and diuretic in treatment of essential hypertension. Biofeedback Selfregulation 1992;17(2):125-141.

Kallinke D, Kulick B, Heim P: Psychologische Behandlungsmöglichkeiten bei essentiellen Hypertonikern; in Köhle K (Hrsg): Zur Psychosomatik von Herz-Kreislauf-Erkrankungen. Berlin, Springer, 1982, pp 775-793. Köhler T: Psychosomatische Krankheiten. Stuttgart, Kohlhammer, 1989.

Latha A, Kaliappan KV: Yoga, pranayama, thermal biofeedback techniques in the management of stress and high blood pressure. J Indian Psychol 1991;9(12):36-46.

Luborsky L, Crits-Christoph P, Brady JP, Kron RE, Weiss T, Cohen M, Levy L: Behavioral versus pharmacological treatments for essential hypertension - a needed comparison. Psychosom Med 1982;44:203-213.

Marwitz M: Ein neuer Weg zur Behandlung der essentiellen Hypertonie: Integrative Biofeedback-Therapie; in Rief W, Birbaumer N (Hrsg): Biofeedback-Therapie. Grundlagen, Indikation und praktisches Vorgehen. Stuttgart, Schattauer, 2000, pp 42-67.

McGrady A: Effects of group relaxation training and thermal biofeedback on blood pressure and related physiological and psychological variables in essential hypertension. Biofeedback Selfregulation 1994;19(1): 51-66.

McGrady A: Good news - bad press: applied psychophysiology in cardiovascular disorders. Biofeedback Selfregulation 1996;21:335-346.

McGrady A: A commentary on 'Problems inherent in assessing biofeedback efficacy studies'. Appl Psychophysiol Biofeedback 2002;27(1):111-112.

McGrady A, Higgins JT Jr: Prediction of response to biofeedback-assisted relaxation in hypertensives: Development of a Hypertensive Predictor Profile (HYPP). Psychosom Med 1989;51:277-284.
McGrady A, Nadsady PA, Schuhmann-Brzezinski C: Sustained effects of biofeedback-assisted relaxation therapy in essential hypertension. Biofeedback Selfregulation 1991;16(4):399-411.

Meichenbaum D: Cognitive factors in biofeedback therapy. Biofeedback Selfregulation 1976;1:201-216.

Musso A, Blanchard EB: Evaluation of thermal biofeedback treatment of hypertension using 24-hr ambulatory blood pressure monitoring. Behav Res Ther 1991;29(5):469-478.

Ohm D: Progressive Relaxation: Tiefmuskelentspannung nach Jacobson. Stuttgart, Trias, 1992.

Piesbergen C, Middeke M, Butollo W: On-line-Feedback des Blutdrucks mittels nichtinvasiver, kontinuierlicher Blutdruckmessung. Nieren Hochdruckkrankheiten 1995;24(3):154-156.

Piesbergen C, Middeke M, Butollo W: Diagnostik und Therapie von Bluthochdruck durch kontinuierliche Messung und Rückmeldung. Hypnose Kogn 1996;13 (1):211-223.

Rief W, Heuser J, Fichter M: Biofeedback - ein therapeutischer Ansatz zwischen Begeisterung und Ablehnung. Verhaltenstherapie 1996;6(1):43-50.

Schandry R: Lebensqualität und Hypertonie. Prävention Rehabil 1994;6(4):174-184.

Schandry R: Lehrbuch der Psychophysiologie. München, PVU, 1996.

Vaitl D: Psychological management of essential hypertension. Contrib Nephrol 1982;30:87-91

Vaitl D: Herzkreislauferkrankungen; in Petermann F Vaitl D (Hrsg): Handbuch der Entspannungsverfahren, vol 2. Weinheim, Beltz, 1994, pp 106-130.

Vickers AJ, Altman DG: Analysing controlled trials with baseline and follow up measurements. BMJ 2001;323:1123-1124.

Waschulewski-Floruß H, Miltner W, Haag G: Biofeedback; in Linden M, Hautzinger M (Hrsg): Verhaltenstherapie. Berlin, Springer, 1994, pp 99-105.

Weiss SM: Biobehavioral approaches to the treatment of hypertension. J S C Med Assoc 1980;9(suppl):38-43. Wittrock DA, Blanchard EB: Thermal biofeedback treatment of mild hypertension: A comparison of effects on conventional and ambulatory blood pressure measures. Behav Modif 1992;16 (3):283-304.

Yucha CB: Problems inherent in assessing biofeedback efficacy studies. Appl Psychophysiol Biofeedback 2002a;27(1):99-106.

Yucha CB: Final remarks. Appl Psychophysiol Biofeedback 2002b;27(1):113-114.

Yucha CB, Clark L, Smith M, Uris P, LaFleur B, Duval S: The effect of biofeedback in hypertension. Appl Nurs Res 2001;14(1):29-35.

Zorn EA, Wilson MB, Angel JJ, Zanella J, Alpert BS: Validation of an automated arterial tonometry monitor using Association for the Advancement of Medical Instrumentation standards. Blood Press Monit 1997;2 3-4. 